\title{
Low-Power Scan Testing and Test Data Compression for System-on-a-Chip
}

\author{
Anshuman Chandra, Student Member, IEEE, and Krishnendu Chakrabarty, Senior Member, IEEE
}

\begin{abstract}
Test data volume and power consumption for scan vectors are two major problems in system-on-a-chip testing. Since static compaction of scan vectors invariably leads to higher power for scan testing, the conflicting goals of low-power scan testing and reduced test data volume appear to be irreconcilable. We tackle this problem by using test data compression to reduce both test data volume and scan power. In particular, we show that Golomb coding of precomputed test sets leads to significant savings in peak and average power, without requiring either a slower scan clock or blocking logic in the scan cells. We also improve upon prior work on Golomb coding by showing that a separate cyclical scan register is not necessary for pattern decompression. Experimental results for the larger ISCAS 89 benchmarks show that reduced test data volume and low power scan testing can indeed be achieved in all cases.
\end{abstract}

Index Terms-Embedded core testing, Golomb codes, power reduction, precomputed test sets, scan testing, switching activity, test set encoding.

\section{INTRODUCTION}

$\mathbf{P}$ REDESIGNED intellectual property (IP) cores are now commonly used in large system-on-a-chip (SOC) designs [1]. An SOC design integrates multiple cores (e.g., microprocessor, memory, DSPs, and I/O controllers) on a single piece of silicon. Despite these benefits, IP cores pose several difficult test challenges. Two problems that are becoming increasingly important are power consumption during manufacturing test and test data volume. The precomputed test patterns provided by the core vendor must be applied to each core within the power constraints of the SOC. In addition, test data compression is necessary to overcome the limitations of the automatic test equipment (ATE), e.g., tester data memory and I/O channel capacity.

Power consumption during testing is important since excessive heat dissipation can damage the circuit under test. Since power consumption in test mode is higher than during normal operation, special care must be taken to ensure that the power rating of the SOC is not exceeded during test application [2]. A number of techniques to control power consumption in test mode have been presented in the literature. These include test scheduling algorithms under power constraints [3], low-power

Manuscript received April 21, 2001; revised September 13, 2001 and January 15,2002 . This work was supported in part by the National Science Foundation under Grant CCR-9875324 and in part by an equipment grant from Intel Corporation. This paper was presented in part at the Design Automation Conference, pp. 166-169, Las Vegas, NV, June 2001. This paper was recommended by Associate Editor K.-T. Cheng.

The authors are with the Department of Electrical and Computer Engineering, Duke University, Durham, NC 27708 USA (e-mail: achandra@ee.duke.edu).

Publisher Item Identifier S 0278-0070(02)02846-4. built-in self-test (BIST) [4]-[7], and techniques for minimizing power during scan testing [8]-[10]. Power consumption and the resulting heat dissipation are especially important for SOCs since test scheduling techniques and test access architectures for system integration attempt to reduce testing time by applying scan/BIST vectors to several cores simultaneously [11]-[15]. Therefore, it is extremely important to decrease power consumption while testing the IP cores in an SOC.

Test data volume is another problem faced in SOC test integration. One way to alleviate this problem is to use BIST. However, BIST can only be applied to SOCs if the IP cores in them are BIST-ready. Since most currently available IP cores are not BIST-ready, the incorporation of BIST in them requires considerable redesign. Hence, test data compression techniques that facilitate low-power scan testing are desirable for SOC testing.

The conflicting goals of low-power scan testing and reduced test data volume appear to be irreconcilable. Test generation for low-power scan testing usually leads to an increase in the number of test vectors [8]. On the other hand, static compaction of scan vectors causes significant increase in power consumption during testing [10]. The compacted vectors are rendered useless if they exceed power constraints. Clearly, uncompacted vectors cannot be used since they require excessive tester memory. This problem is addressed in a recent paper on power-constrained static compaction of scan vectors [10]. However, while [10] provides 2-3 times reduction in power consumption for several ISCAS benchmark circuits, it does not lead to any appreciable reduction in test data volume-in fact, it does not provide any improvement over standard static vector compaction techniques. Furthermore, the scheme presented in [10] only addresses scan-in power and it does not consider power dissipation during the scan-out operation.

Recently, a number of data compression techniques have been proposed for reducing SOC test data volume [17]-[24]. In this approach, the precomputed test set $T_{D}$ provided by the core vendor is compressed (encoded) to a much smaller test set $T_{E}$ and stored in ATE memory; see Fig. 1. An on-chip decoder is used for pattern decompression to generate $T_{D}$ from $T_{E}$ during pattern application. It was shown in [19], [20], and [24] that compressing a "difference vector" sequence $T_{\text {diff }}$ determined from $T_{D}$ results in smaller test sets and reduces testing time. Fig. 2 shows the test architecture based on $T_{\text {diff }}$ and a cyclical scan registers (CSR). An obvious drawback of this approach is that it requires a separate CSR.

In this paper, we dispel the notion that scan vector compaction always leads to higher power consumption. Since static compaction invariably leads to higher power, we explore test data compression for overcoming this problem. We show that test 


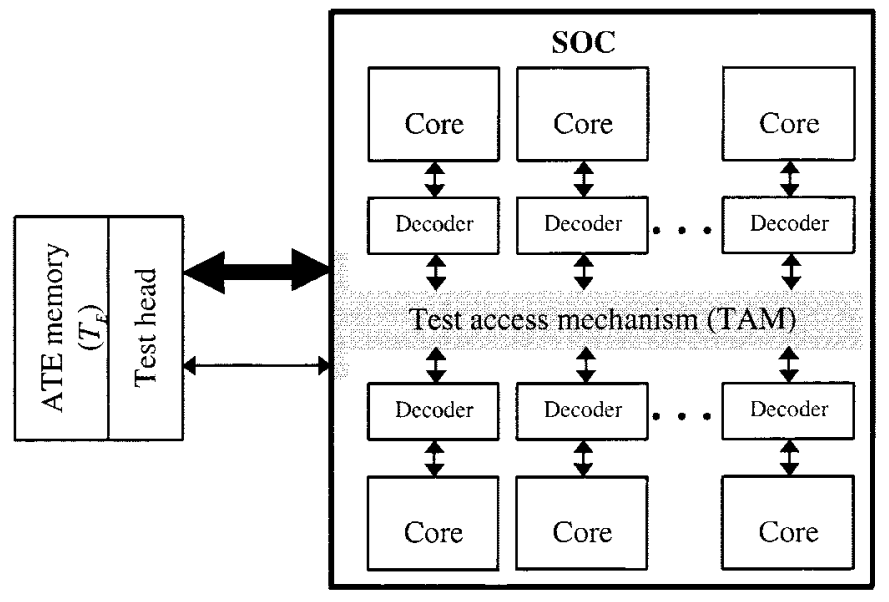

Fig. 1. A conceptual architecture for testing a system-on-chip by storing the encoded test data $T_{E}$ in ATE memory and decoding it using on-chip decoders.

data compression leads to significant reduction in power consumption during scan testing. In particular, we show that we can decrease both peak and average power by using Golomb codes for compressing the scan vectors of IP cores. In this way, there is no need to either reduce the scan clock rate for low power or add blocking logic to the scan cells [5]. The use of a low-cost on-chip decoder allows us to achieve significant test data compression, and the decompressed scan vectors cause very little switching activity in the scan chains during test application. While we only target scan-in power in our compression scheme, we show experimentally that significant savings are also obtained in scan-out power. In addition, we show that it is not necessary to use a separate CSR; we can directly encode $T_{D}$ and thereby obviate the need for the CSR (Fig. 3).

The organization of the paper is as follows. In Section II, we first review Golomb coding. We then describe the data compression procedure and the decompression architecture and highlight the key differences from [20]. Section III shows how we can combine low-power scan testing with test data compression. Finally, in Section IV we present experimental results for the large ISCAS 89 benchmark circuits as well as for a real-life microprocessor circuit from IBM.

\section{COMPRESSION MEthOD AND TEST ARCHITECTURE}

We first review Golomb coding and its application to test data compression in [20]. The major advantages of Golomb coding of test data include very high compression, analytically predictable compression results, and a low-cost and scalable on-chip decoder. In addition, the novel interleaving decompression architecture allows multiple cores in an SOC to be tested concurrently using a single ATE I/O channel.

If the difference vector $T_{\text {diff }}$ is used for compression, the first step is to derive it from $T_{D}$, where $T_{D}=\left\{t_{1}, t_{2}, t_{3}, \ldots, t_{n}\right\}$ is the (ordered) precomputed test set. The ordering is determined using a heuristic procedure described later. $T_{\text {diff }}$ is defined as follows:

$$
\begin{aligned}
T_{\text {diff }} & =\left\{d_{1}, d_{2}, \ldots, d_{n}\right\} \\
& =\left\{t_{1}, t_{1} \oplus t_{2}, t_{2} \oplus t_{3}, \ldots, t_{n-1} \oplus t_{n}\right\}
\end{aligned}
$$

where a bit-wise exclusive-or operation is carried out between patterns $t_{i}$ and $t_{j}$. This assumes that the CSR starts in the all-0 state. (Other starting states can be considered similarly.)

In this work, however, we encode $T_{D}$ directly, hence there is no need to generate $T_{\text {diff. }}$. All the don't care bits in $T_{D}$ are mapped to zeros to obtain a fully-specified test sequence. We show in Section IV that better compression is obtained using $T_{D}$ instead of $T_{\text {diff }}$ in almost all cases.

The next step in the encoding procedure is to select the Golomb code parameter $m$, referred to as the group size [21]. Once $m$ is determined, e.g., using the methods described in [21], the runs of zeros in the test data stream are mapped to groups of size $m$ (each group corresponding to a run length). The number of such groups is determined by the length of the longest run of zeros in $T_{D}$. The set of run-lengths $\{0,1,2, \ldots, m-1\}$ forms group $A_{1}$; the set $\{m, m+1, m+2, \ldots, 2 m-1\}$, group $A_{2}$; etc. In general, the set of run-lengths $\{(k-1) m,(k-1) m+$ $1,(k-1) m+2, \ldots, k m-1\}$ comprises group $A_{k}$. To each group $A_{k}$, we assign a group prefix of $(k-1)$ ones followed by a zero. We denote this by $1^{(k-1)} 0$. If $m$ is chosen to be a power of 2 i.e., $m=2^{N}$, each group contains $2^{N}$ members and a $\log _{2} m$-bit sequence (tail) uniquely identifies each member within the group. Thus, the final code word for a run-length $L$ that belongs to group $A_{k}$ is composed of two parts: a group prefix and tail. The prefix is $1^{(k-1)} 0$ and the tail is a sequence of $\log _{2} m$ bits. The encoding process is illustrated in Table I for $m=4$.

Scan vectors can be reordered to decrease test data volume. The problem of determining the best ordering of test vectors is equivalent to the NP-complete traveling salesman problem. Therefore, a greedy algorithm is used to generate an ordering and the corresponding $T_{D}$. Suppose a partial ordering $t_{1} t_{2} \cdots t_{i}$ has already been determined for the patterns in $T_{D}$. To determine $t_{i+1}$, we calculate the Hamming distance $H D\left(t_{i}, t_{j}\right)$ between $t_{i}$ and all patterns $t_{j}$ that have not been placed in the ordered list. We define $H D\left(t_{i}, t_{j}\right)$ as the number of zeros in the pattern $t_{j}$. This metric is chosen since it tends to produce longer runs of zeros. We select the pattern $t_{j}$ for which $H D\left(t_{i}, t_{j}\right)$ is maximum and add it to the ordered list, denoting it by $t_{i+1}$. In this way, a fully specified test pattern is obtained and the smallest number of ones is added to the ordered vector sequence. We continue this process until all test patterns in $T_{D}$ are placed in the ordered list. Fig. 4 illustrates the procedure for obtaining fully specified ordered $T_{D}$.

An on-chip decoder decompresses the encoded test set $T_{E}$ and produces $T_{D}$. Even though $T_{D}$ contains more patterns than test sets obtained after static compaction of ATPG vectors, the testing time is reduced since pattern decompression can be carried out on-chip at higher clock frequencies. As discussed in [20], the decoder can be efficiently implemented by a $\log _{2} m$-bit counter and a finite-state machine (FSM) and is independent of the precomputed test set and the circuit under test. The block diagram of the decoder for $m=4$ is shown in Fig. 5. The synthesized decode FSM circuit contains only four flip-flops and 34 combinational gates. For any circuit whose test set is compressed using $m=4$, the given logic is the only additional hardware required other than the two-bit counter. 


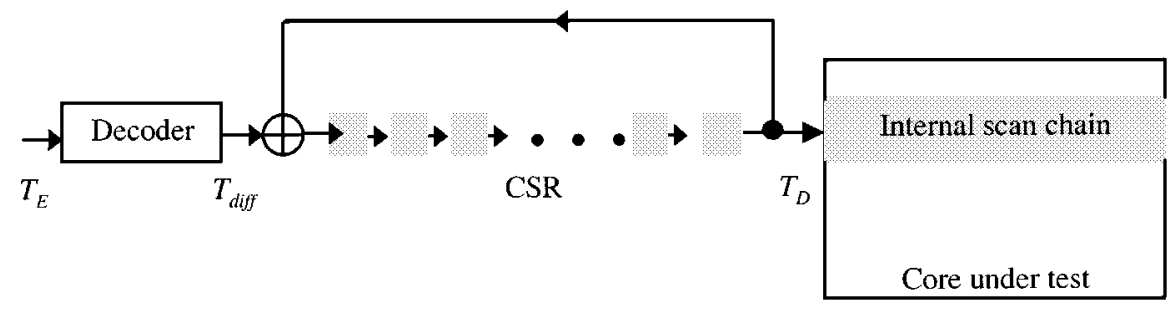

Fig. 2. Decompression architecture based on a CSR.

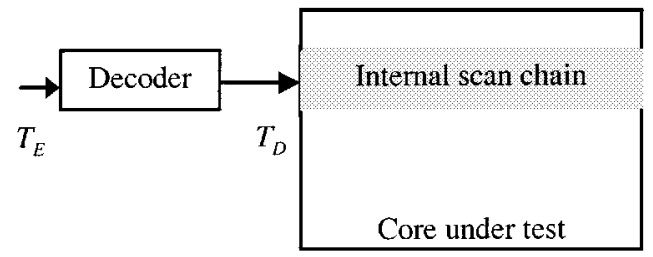

Fig. 3. Test architecture based on Golomb coding of the precomputed test set $T_{D}$.

TABLE I

EXAMPLE OF GOLOMB CODING FOR $m=4$

\begin{tabular}{|c|c|c|c|c|}
\hline Group & Run-length & $\begin{array}{l}\text { Group } \\
\text { prefix }\end{array}$ & Tail & Codeword \\
\hline \multirow{4}{*}{$A_{1}$} & 0 & \multirow{4}{*}{0} & 00 & 000 \\
\hline & 1 & & 01 & 001 \\
\hline & 2 & & 10 & 010 \\
\hline & 3 & & 11 & 011 \\
\hline \multirow{4}{*}{$A_{2}$} & 4 & \multirow{4}{*}{10} & 00 & 1000 \\
\hline & 5 & & 01 & 1001 \\
\hline & 6 & & 10 & 1010 \\
\hline & 7 & & 11 & 1011 \\
\hline \multirow{4}{*}{$A_{3}$} & 8 & \multirow{4}{*}{110} & 00 & 11000 \\
\hline & 9 & & 01 & 11001 \\
\hline & 10 & & 10 & 11010 \\
\hline & 11 & & 11 & 11011 \\
\hline$\ldots$ & $\ldots$ & $\ldots$ & $\cdots$ & $\cdots$ \\
\hline
\end{tabular}

This is especially the case if, unlike in [20], $T_{D}$ is directly used for encoding and a CSR is not required for decompression.

Since the decoder for Golomb coding needs to communicate with the tester, and both the codewords and the decompressed data can be of variable length, proper synchronization must be ensured through careful design. In particular, the decoder must communicate with the tester to signal the end of a block of variable-length decompressed data. These and other related decompression issues are discussed in detail in [20].

\section{Power ESTIMATION FOR ScAn Vectors}

In this section, we examine the impact of test set encoding on power consumption during scan testing. We then show how power consumption can be minimized by appropriately assigning binary values to the don't care bits in $T_{D}$ and then applying Golomb coding for test data compression.

For a CMOS circuit, power consumption can be classified as either static or dynamic. Static power consumption, which is caused by leakage current, is usually negligible and therefore ignored. Dynamic power is consumed when the outputs of circuit elements make high-to-low and low-to-high transitions.

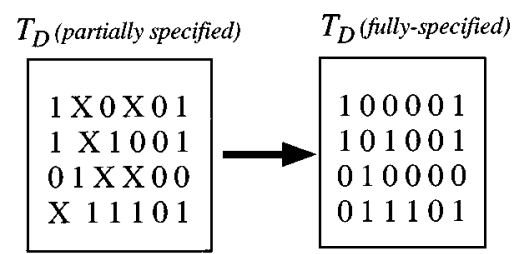

$T_{D}$

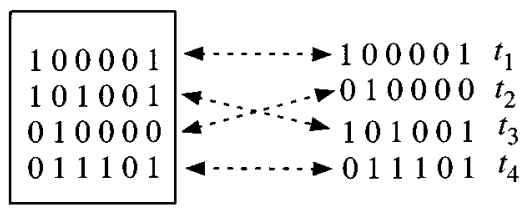

$T_{D}=\left\{t_{1}, t_{2}, t_{3}, t_{4}\right\}=\{100001,010000,101001,011101\}$

Fig. 4. An example to illustrate the procedure of deriving fully specified ordered $T_{D}$.

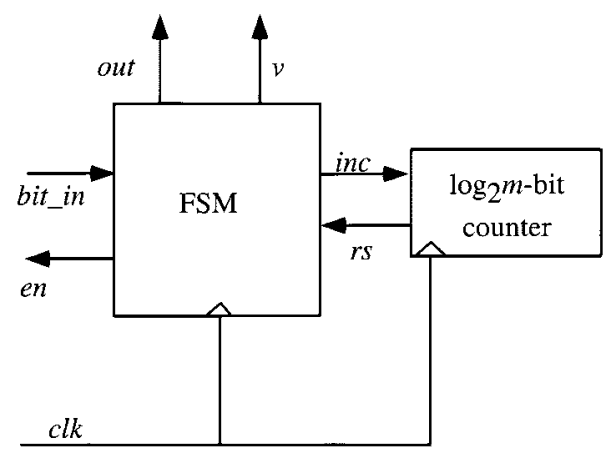

Fig. 5. The decoder block diagram for Golomb code parameter $m=4$ [20].

This constitutes the predominant fraction of CMOS power consumption.

For scan vectors, the dynamic power consumption during testing depends on the number of transitions that occur in the scan chain as well as on the number of circuit elements that switch during the scan in and scan out operations. Power estimation models based on the switching activity of circuits have been presented in the literature [6], [10]. We use the weighted transitions metric (WTM) introduced in [10] to estimate the power consumption due to scan vectors. This model was validated in [10], hence we do not report on its accuracy in this paper. The WTM metric models the fact that the scan in power for a given vector depends not only on the number of transitions in it but also on their relative positions. For example, consider a scan vector $v_{1} v_{2} v_{3} v_{4} v_{5}=01000$, where $v_{1}$ is first loaded into the scan chain. The 0-to-1 transition between $v_{1}$ and $v_{2}$ causes more switching activity in the scan chain than 
TABLE II

MAPPING OF DON'T CARES IN $T_{D}$ TO BINARY VALUES

\begin{tabular}{c|c|c}
\hline $\begin{array}{c}\text { Partially-specified } \\
\text { scan vector }\end{array}$ & $\begin{array}{c}\text { Fully-specified vector } \\
\text { (Minimum } W T M)\end{array}$ & $\begin{array}{c}\text { Fully-specified vector } \\
\text { (Don't-cares mapped to 0s) }\end{array}$ \\
\hline$t_{i}=01 X X X 10 X X X 01$ & 011111000001 & 010001000001 \\
& Golomb code length: 19 bits & Golomb code length: 10 bits \\
& $W T=4)$ & $W=4)$ \\
\hline$t_{j}=01 X 1010 X X X X 1$ & 011101011111 & $W T M_{i}=25$ \\
& Golomb code length: 27 bits & Golomb code length: 13 bits \\
& $(m=4)$ & $(m=4)$ \\
& $W T M_{j}=23$ & $W T M_{j}=32$ \\
\hline
\end{tabular}

the 1-to-0 transition between $v_{2}$ and $v_{3}$. We use the same model to estimate the power consumption during scan out operation.

The weighted transitions count metric is also strongly correlated to the switching activity in the internal nodes of the core under test during the scan in operation. It was shown experimentally in [10] that scan vectors that have higher weighted transition metric dissipate more power in the core under test.

Consider a scan chain of length $l$ and a scan vector $t_{j}=$ $t_{j, 1}^{\star} t_{j, 2}^{\star} \cdots t_{j, l}^{\star}$, with $t_{j, 1}^{\star}$ scanned in before $t_{j, 2}^{\star}$, and so on. The weighted transitions metric for $t_{j}$, denoted $W T M_{j}$, is given by $W T M_{j}=\sum_{i=1}^{l-1}(l-i) \cdot\left(t_{j, i}^{\star} \oplus t_{j, i+1}^{\star}\right)$. If the test set $T_{D}$ contains $n$ vectors $t_{1}, t_{2}, \ldots, t_{n}$, then the average scan in power $P_{\text {avg }}$ and peak scan in power $P_{\text {peak }}$ are estimated as follows:

$$
\begin{gathered}
P_{\text {avg }}=\frac{\sum_{j=1}^{n} \sum_{i=1}^{l-1}(l-i) \cdot\left(t_{j, i}^{\star} \oplus t_{j, i+1}^{\star}\right)}{n} \\
P_{\text {peak }}=\max _{j \in\{1,2, \ldots, n\}}\left\{\sum_{i=1}^{l-1}(l-i) \cdot\left(t_{j, i}^{\star} \oplus t_{j, i+1}^{\star}\right)\right\} .
\end{gathered}
$$

If the peak power exceeds a threshold value, it can cause structural damage to the silicon or to the package. Likewise, elevated average power can also cause structural damage to the silicon, bonding wires, or the package. It also adds to the thermal load that must be transported away from the device under test.

We next show how Golomb codes can be used to minimize the volume of test data and, at the same time, minimize $P_{\text {avg }}$ and $P_{\text {peak }}$. Scan-in power is influenced by the manner in which the don't cares in $T_{D}$ are mapped to binary values. While $P_{\text {avg }}$ and $P_{\text {peak }}$ can be minimized by choosing an appropriate mapping, such a mapping is not guaranteed to provide high test data compression. In fact, our experiments show that the encoded test sets in such cases are often larger than the uncompacted test sets. Instead, it is far more efficient to simply map all the don't cares in $T_{D}$ to zeros as shown in Fig. 4. While this approach does not minimize $P_{\text {avg }}$ and $P_{\text {peak }}$, it provides significant reductions in power consumption and, at the same time, decreases the test data volume considerably. The fully specified test set thus obtained is then compressed using Golomb codes. Since uncompacted test cubes contain a large number of don't cares, mapping these don't cares to zeros results in long runs of zeros. These long runs of zeros provide very high test data compression as well as reduced transitions during scan in. Even though we do not directly address scan-out power, our experiments with benchmark circuits show that this approach reduces the number of transitions and the resulting WTM during scan out. This is an added advantage of using encoded test sets for scan testing.

For example, Table II shows two partially specified scan vectors $t_{i}=01---10---01$ and $t_{j}=01-1010----1$ with scan chain length $l=12$, where - denotes a don't care bit. If the don't cares are mapped to binary values to minimize the weighted transition metric, then $d----d^{\prime}, d \in\{0,1\}$ must be mapped to $d d d d d d^{\prime}$. Similarly, $d----$ must be mapped to $d d d d d$. This ensures that the few unavoidable transitions occur "late" during scan in. Table II shows the values of $W T M_{i}$ and $W T M_{j}$ and the Golomb codes for the corresponding fully specified vectors $(m=4)$. The weighted transitions metric is clearly higher if the don't cares are always mapped to zero. However, Golomb coding is much more effective in reducing test data volume if this strategy is used. We show in the next section that mapping don't cares to zeros reduces test data volume considerably without any significant decrease in power savings.

First, we derive the following theorem which characterizes the maximum WTM for a given test length $n$, scan chain length $l$, and the number of ones $r$ in the test set. This yields the maximum value for the average power $P_{\text {avg }}$ and it can be used to predict average power by using limited information about $T_{D}$. We use this theorem in Section IV to derive the upper bound on $P_{\text {avg }}$ for ISCAS 89 benchmark circuits. The maximum value for the peak power is obtained for a test pattern that has alternating ones and zeros and thus has the maximum switching activity. Hence peak power $P_{\text {peak }}$ is given by

$$
\begin{aligned}
P_{\text {peak }} & =(l-1)+(l-2)+(l-3)+\cdots+1 \\
& =\frac{l(l-1)}{2}
\end{aligned}
$$

as long as $r \geq l / 2$.

Theorem 1: For a given test length $n$, scan chain length $l$, and the number of ones $r$ in the test set, the average power is given by

$$
P_{\text {avg }} \leq \frac{l r}{n}-\frac{r^{2}}{n^{2}}+\frac{r}{2 n^{3}}\left(\frac{r}{n}+1\right) .
$$

Proof: Let $r=Q n+R$ such that $Q=\lfloor r / n\rfloor$. The WTM for the entire test set is maximum when the ones are distributed 
TABLE III

EXPERIMENTAL RESUlTS ON TEST DATA COMPRESSION USING GOLOMB CODES

\begin{tabular}{|c|c|c|c|c|c|c|c|}
\hline \multirow[b]{2}{*}{ Circuit } & \multirow[b]{2}{*}{$\begin{array}{c}\text { No. of } \\
\text { bits in } \\
T_{D}\end{array}$} & \multirow[b]{2}{*}{$\begin{array}{c}\text { Size of } \\
\text { Mintest test } \\
\text { set (bits) }\end{array}$} & \multicolumn{2}{|c|}{ Golomb coding using $T_{\text {diff }}$} & \multicolumn{3}{|c|}{ Golomb coding using $T_{D}$} \\
\hline & & & $\begin{array}{c}\text { Comp- } \\
\text { pression } \\
\text { (percent) }\end{array}$ & $\begin{array}{l}\text { No. of } \\
\text { bits } \\
\text { in } T_{E}\end{array}$ & $\begin{array}{l}\text { Comp- } \\
\text { pression } \\
\text { (percent) }\end{array}$ & $\begin{array}{c}\text { No. of } \\
\text { bits } \\
\text { in } T_{E}\end{array}$ & $\begin{array}{l}\text { Improvement } \\
\text { over Mintest } \\
\text { (percent) }\end{array}$ \\
\hline s5378 & 23754 & 20758 & $40.70(m=4)$ & 14085 & $37.11(m=4)$ & 14937 & 28.04 \\
\hline s9234 & 39273 & 25935 & $43.34(m=4)$ & 22250 & $45.25(m=4)$ & 21499 & 17.10 \\
\hline s13207 & 165200 & 163100 & $74.78(m=16)$ & 41658 & $79.74(m=16)$ & 33467 & 79.48 \\
\hline$s 15850$ & 76986 & 57434 & $47.11(m=4)$ & 40717 & $62.82(m=8)$ & 28618 & 50.17 \\
\hline s38417 & 164736 & 113152 & $44.12(m=4)$ & 92054 & $28.37(m=4)$ & 117987 & -4.27 \\
\hline s38584 & 199104 & 161040 & $47.71(m=4)$ & 104111 & $57.17(m=8)$ & 85275 & 47.05 \\
\hline Average & - & - & 49.63 & - & 51.74 & - & 36.26 \\
\hline
\end{tabular}

in alternating fashion as discussed above. Hence, the maximum WTM is given by

$$
\begin{aligned}
\max & W T M \\
& =\ln +(l-1) n+\cdots+(l-Q+1) n+(l-Q) R \\
& =\left(Q l-\frac{Q(Q-1)}{2}\right) n+(l-Q) R \\
& =l r-\left\lfloor\frac{r^{2}}{n}\right\rfloor+\frac{1}{2 n}\left\lfloor\frac{r}{2 n}\right\rfloor\left(\left\lfloor\frac{r}{n}\right\rfloor+1\right) \\
& \leq l r-\frac{r^{2}}{n}+\frac{r}{2 n^{2}}\left(\frac{r}{n}+1\right) .
\end{aligned}
$$

The maximum average power is now obtained by dividing $\max W T M$ by $n$.

\section{EXPERIMENTAL RESULTS}

In this section, we evaluate the effect of Golomb coding of $T_{D}$ on test data volume and power consumption during scan testing for the ISCAS 89 benchmark circuits. The experiments were conducted on a Sun Ultra 10 workstation with a $333 \mathrm{MHz}$ processor and $256 \mathrm{MB}$ of memory. We only considered the large full-scan circuits with a single scan chain each. The test vectors for these circuits were reordered to increase compression. The amount of compression obtained was computed as follows:

\section{Compression (percent)

$$
=\frac{\text { (Total no. bits in } \left.T_{D}-\text { Total no. bits in } T_{E}\right)}{\left(\text { Total no. bits in } T_{D}\right)} \times 100 \text {. }
$$

Table III presents the experimental results for test cubes $T_{D}$ obtained from the Mintest ATPG program with dynamic compaction [25]. In order to compare with [20], we also present compressed results obtained using the difference vector sequences $T_{\text {diff }}$ for the same test sets. The table lists the sizes of the precomputed (uncompacted) test sets, the amount of compression achieved for the best value of the Golomb code $m$, and the size of the smallest encoded test set obtained after static compaction using Mintest.

As is evident from Table III, $T_{D}$ yields better compression than $T_{\text {diff }}$ in four out of the six cases. For these circuits, we achieve better compression without requiring a separate CSR. (The best value of the code parameter $m$ is shown in paren- thesis.) Therefore, there is a significant reduction in hardware overhead as compared to the compression scheme presented in [19] and [20]. The results also show that ATPG compaction may not always be necessary for saving memory and reducing testing time. In five out of the six cases, the size of the encoded test set is less than the smallest ATPG-compacted test sets known for these circuits. This comparison is essential in order to show that storing $T_{E}$ in ATE memory is more efficient than simply applying static compaction to test cubes and storing the resulting compact test sets. On average, the size of $T_{E}$ is $36.26 \%$ less than that of the compacted test sets obtained using Mintest.

We next present results on the peak and average power consumption during the scan-in operation. These results show that test data compression can also lead to significant savings in power consumption. As described in Section III, we estimate power using the weighted transitions metric. Let $P_{\text {peak }}^{C}\left(P_{\text {avg }}^{C}\right)$ be the peak (average) power with compacted test sets obtained using Mintest. Similarly, let $P_{\text {peak }}^{G}\left(P_{\text {avg }}^{G}\right.$ ) be the peak (average) power when Golomb coding is used by mapping the don't cares in $T_{D}$ to zeros. Table IV compares the average and peak power consumption for Mintest test sets with $T_{D}$ when Golomb coding is used. The percentage reduction in power was computed as follows:

Reduction in peak power consumption

$$
=\frac{P_{\mathrm{peak}}^{C}-P_{\mathrm{peak}}^{G}}{P_{\mathrm{peak}}^{C}} \times 100
$$

Reduction in average power consumption

$$
=\frac{P_{\mathrm{avg}}^{C}-P_{\mathrm{avg}}^{G}}{P_{\mathrm{avg}}^{C}} \times 100 .
$$

Table IV shows that the peak power and average power are significantly less if Golomb coding is used for test data compression and the decompressed patterns are applied during testing. On average, the peak (average) power is $28.98 \%$ $(75.89 \%)$ less in this case than for the Mintest test sets.

We next present results on the peak and average power consumption during the scan-out operation. Table $\mathrm{V}$ shows that the peak power and average power are significantly less if Golomb coding is used for test data compression. On average, the peak (average) power is $23.54 \%$ (57.31\%) less than for the Mintest test sets. Thus, our results demonstrate that the substantial reduction in test data volume is also accompanied by significant 
TABLE IV

EXPERIMENTAL RESUlts ON PEAK AND Average SCAN-In POWER CONSUMPTION

\begin{tabular}{c|c|c|c|c|c|c}
\hline & \multicolumn{2}{|c|}{$\begin{array}{c}\text { Mintest test sets } \\
\text { after static compaction }\end{array}$} & \multicolumn{4}{c}{$\begin{array}{c}\text { Uncompacted } \\
\text { test sets }\end{array}$} \\
\cline { 2 - 7 } Circuit & $\begin{array}{c}\text { Peak } \\
\text { power }\end{array}$ & $\begin{array}{c}\text { Average } \\
\text { power } \\
\end{array}$ & $\begin{array}{c}\text { Peak } \\
\text { power }\end{array}$ & $\begin{array}{c}\text { Peak power } \\
\text { reduction } \\
\text { (percent) }\end{array}$ & $\begin{array}{c}\text { Average } \\
\text { power } \\
P_{a v g}^{G}\end{array}$ & $\begin{array}{c}\text { Average power } \\
\text { reduction } \\
\text { (percent) }\end{array}$ \\
\hline s5378 & 13423 & 11081 & 10127 & 24.55 & 3336 & 69.89 \\
s9234 & 17494 & 14630 & 12994 & 25.72 & 5692 & 61.09 \\
s13207 & 135607 & 122031 & 101127 & 25.42 & 12416 & 89.82 \\
s15850 & 100228 & 90899 & 81832 & 18.35 & 20742 & 77.18 \\
s35932 & 707280 & 583639 & 172834 & 75.56 & 73080 & 87.47 \\
s38417 & 683765 & 601840 & 505295 & 26.10 & 172665 & 71.31 \\
s38584 & 572618 & 535875 & 531321 & 7.21 & 136634 & 74.50 \\
\hline Average & - & - & - & 28.98 & - & 75.89 \\
\hline
\end{tabular}

TABLE V

EXPERIMENTAL RESUltS ON PEAK AND AVERAGe SCAN-OUt POWER CONSUMPTION

\begin{tabular}{|c|c|c|c|c|c|c|}
\hline \multirow[b]{2}{*}{ Circuit } & \multicolumn{2}{|c|}{$\begin{array}{c}\text { Mintest test sets } \\
\text { after static compaction }\end{array}$} & \multicolumn{4}{|c|}{$\begin{array}{l}\text { Uncompacted } \\
\text { test sets }\end{array}$} \\
\hline & $\begin{array}{l}\text { Peak } \\
\text { power } \\
P_{\text {peak }}^{C}\end{array}$ & $\begin{array}{c}\text { Average } \\
\text { power } \\
P_{a v g}^{C}\end{array}$ & $\begin{array}{c}\text { Peak } \\
\text { power } \\
P_{\text {peak }}^{G}\end{array}$ & $\begin{array}{l}\text { Peak power } \\
\text { reduction } \\
\text { (percent) }\end{array}$ & $\begin{array}{c}\text { Average } \\
\text { power } \\
P_{a v g}^{G}\end{array}$ & $\begin{array}{l}\text { Average power } \\
\text { reduction } \\
\text { (percent) }\end{array}$ \\
\hline s9234 & 16777 & 14555 & $\frac{p e a n}{13855}$ & 17.41 & 9218 & 36.66 \\
\hline s13207 & 132129 & 116695 & 116644 & 11.72 & 39816 & 65.88 \\
\hline s 15850 & 99647 & 88385 & 83914 & 15.78 & 32972 & 62.69 \\
\hline s35932 & 745282 & 390622 & 145163 & 80.52 & 53520 & 86.29 \\
\hline s 38417 & 619929 & 553491 & 572593 & 7.63 & 294266 & 46.83 \\
\hline s38584 & 577365 & 521882 & 530042 & 8.19 & 284135 & 45.55 \\
\hline Average & - & - & - & 23.54 & - & 57.31 \\
\hline
\end{tabular}

TABLE VI

IMPACT OF THE MAPPING OF DON'T CARES to BinaRY VALUES ON POWER CONSUMPTION

\begin{tabular}{|c|c|c|c|c|c|c|c|c|}
\hline \multirow[b]{2}{*}{ Circuit } & \multicolumn{4}{|c|}{$\begin{array}{l}\text { Uncompacted test sets } \\
\text { with don't-cares } \\
\text { mapped to 0s }\end{array}$} & \multicolumn{4}{|c|}{$\begin{array}{l}\text { Uncompacted test sets } \\
\text { with don't-cares mapped to } \\
\text { minimize WTM }\end{array}$} \\
\hline & $\begin{array}{c}\text { Peak } \\
\text { power } \\
P_{\text {peak }}^{G} \\
\end{array}$ & $\begin{array}{c}\begin{array}{c}\text { Peak power } \\
\text { reduction } \\
\text { (percent) }\end{array} \\
\end{array}$ & $\begin{array}{c}\text { Average } \\
\text { power } \\
P_{a v g}^{G} \\
\end{array}$ & $\begin{array}{c}\text { Average power } \\
\text { reduction } \\
\text { (percent) }\end{array}$ & $\begin{array}{c}\text { Peak } \\
\text { power } \\
P_{\text {peak }}^{G} \\
\end{array}$ & $\begin{array}{l}\text { Peak power } \\
\text { reduction } \\
\text { (percent) }\end{array}$ & $\begin{array}{c}\text { Average } \\
\text { power } \\
P_{a v g}^{G} \\
\end{array}$ & $\begin{array}{c}\text { Average power } \\
\text { reduction } \\
\text { (percent) } \\
\end{array}$ \\
\hline s5378 & 10127 & 24.55 & 3336 & 69.89 & 9531 & 28.99 & 2435 & 78.02 \\
\hline s9234 & 12994 & 25.72 & 5692 & 61.09 & 12060 & 31.06 & 3466 & 76.30 \\
\hline s13207 & 101127 & 25.42 & 12416 & 89.82 & 97606 & 28.02 & 7703 & 93.68 \\
\hline s15850 & 81832 & 18.35 & 20742 & 77.18 & 63478 & 36.66 & 13381 & 85.27 \\
\hline s35932 & 172834 & 75.56 & 73080 & 87.47 & 125490 & 82.25 & 46032 & 92.11 \\
\hline s38417 & 505295 & 26.10 & 172665 & 71.31 & 404617 & 40.82 & 112198 & 81.35 \\
\hline s38584 & 531321 & 7.21 & 136634 & 74.50 & 479530 & 16.25 & 88298 & 83.52 \\
\hline Average & - & 28.98 & - & 75.89 & - & 37.72 & - & 84.32 \\
\hline
\end{tabular}

reduction in power consumption during scan testing. The reduction in scan-out power is an important added advantage since we do not directly target scan-out power in our compression scheme.

Next, we justify the strategy of mapping all don't cares in $T_{D}$ to zeros before Golomb coding. As discussed in Section III, the power consumption can be minimized if the don't cares are assigned to binary values to minimize the weighted transitions metric. Unfortunately, this strategy does not lead to any significant decrease in the test data volume - in fact, we found that in many cases, the encoded test set was larger than the original test set. We therefore carried out a set of experiments to demonstrate that if all don't cares are mapped to zeros, the test data volume decreases substantially (Table III), and at the same time power savings is significant.

Our experimental results for the larger ISCAS 89 circuits are shown in Table VI. We note that while the average power consumption is greater compared to the "optimal" mapping of don't-cares, it is still significantly less than the power for ATPGcompacted test sets. In some cases, the difference is as low as $4 \%$, while on average, the average power consumption increases by only $8 \%$. Likewise, the difference in peak power consumption is only $9 \%$ on average. Nevertheless, compared to Mintest, we achieve $51 \%$ test data compression on average with $76 \%$ re- 
TABLE VII

COMPRESSION OBTAINED FOR TEST DATA FROM INDUSTRY

\begin{tabular}{|c|c|c|c|}
\hline \multirow[b]{2}{*}{$\begin{array}{l}\text { Scan } \\
\text { Vector }\end{array}$} & \multirow{2}{*}{$\begin{array}{c}\text { Size of } \\
T_{D} \\
\text { (bits) }\end{array}$} & \multicolumn{2}{|c|}{ Golomb codes } \\
\hline & & $\begin{array}{c}\text { Size of } \\
T_{E}(m=128)\end{array}$ & $\begin{array}{l}\text { Percentage } \\
\text { compression }\end{array}$ \\
\hline 1 & 362922 & 27708 & 92.36 \\
\hline 2 & 362922 & 20406 & 94.37 \\
\hline 3 & 362922 & 20406 & 94.37 \\
\hline 4 & 362922 & 20244 & 94.42 \\
\hline 5 & 362922 & 18267 & 94.96 \\
\hline 6 & 362922 & 14081 & 96.12 \\
\hline 7 & 362922 & 14418 & 96.02 \\
\hline 8 & 362922 & 14370 & 96.04 \\
\hline 9 & 362922 & 12875 & 96.45 \\
\hline 10 & 362922 & 10414 & 97.13 \\
\hline 11 & 362922 & 9729 & 97.31 \\
\hline 12 & 362922 & 11199 & 96.91 \\
\hline 13 & 362922 & 8618 & 97.62 \\
\hline 14 & 362922 & 8675 & 97.60 \\
\hline 15 & 362922 & 8123 & 97.76 \\
\hline 16 & 362922 & 6508 & 98.20 \\
\hline 17 & 362922 & 7629 & 97.89 \\
\hline 18 & 362922 & 7974 & 97.80 \\
\hline 19 & 362922 & 7668 & 97.88 \\
\hline 20 & 362922 & 6791 & 98.12 \\
\hline 21 & 362922 & 8149 & 97.75 \\
\hline 22 & 362922 & 7120 & 98.03 \\
\hline 23 & 362922 & 6959 & 98.08 \\
\hline 24 & 362922 & 5856 & 98.38 \\
\hline 25 & 362922 & 5911 & 98.37 \\
\hline 26 & 362922 & 6254 & 98.27 \\
\hline 27 & 362922 & 6903 & 98.09 \\
\hline 28 & 362922 & 9212 & 97.46 \\
\hline 29 & 362922 & 6460 & 98.22 \\
\hline 30 & 362922 & 5580 & 98.46 \\
\hline 31 & 362922 & 5994 & 98.34 \\
\hline 32 & 362922 & 6118 & 98.31 \\
\hline
\end{tabular}

TABLE VIII

Comparison of UpPer Bound on $P_{\text {avg }}$ (Predicted by THEOREM 1) With the ACtual VALUE of $P_{\text {avg }}$

\begin{tabular}{c|c|c|c|c|c}
\hline Circuit & $l$ & $n$ & $r$ & $\begin{array}{c}P_{a v g} \\
\text { (computed) }\end{array}$ & $\begin{array}{c}\text { Upper bound } \\
\text { on } P_{\text {avg }}\end{array}$ \\
\hline s5378 & 214 & 111 & 3538 & 3336 & 5795 \\
s9234 & 247 & 159 & 4817 & 5692 & 6564 \\
s13207 & 700 & 236 & 5021 & 12416 & 14436 \\
s15850 & 611 & 126 & 5336 & 20742 & 24056 \\
s38417 & 1664 & 99 & 29473 & 172665 & 406749 \\
s38584 & 1464 & 136 & 16814 & 136634 & 165710 \\
\hline
\end{tabular}

duction in average power consumption for scan testing. This provides a strong justification for the proposed test data compression approach.

We next present experimental results for a real test set from industry. We obtained a set of 32 scan vectors from IBM (a total of 362922 bits of test data per vector) for a design with 3.6 million gates and 726000 latches. The compression results for the 32 scan vectors is shown in Table VII. These vectors are statically compacted tests and we mapped the remaining don't-cares to zeros to reduce scan power and increase the amount of com- pression. Note that we obtain a staggering $97.10 \%$ compression on average. We do not have any direct means to compare the WTM measure here with a base case; nevertheless, we expect significant power savings due to the presence of long runs of zeros in the patterns fed to the scan chain after on-chip decompression.

Finally, we compare the upper bound on $P_{\text {avg }}$ provided by Theorem 1 provided with the actual value of $P_{\text {avg }}$ for the ISCAS 89 circuits. Table VIII shows that in most cases, Theorem 1 can be used as a reasonable predictor of average power consumption for a precomputed test set.

\section{CONCLUSION}

We have addressed the problems of test data volume and power consumption for scan vectors for system-on-a-chip testing. Since static compaction of scan vectors invariably leads to higher power for scan testing, the conflicting goals of low-power scan testing and reduced test data volume appear to be irreconcilable. In this paper, we have employed test data compression to tackle these problems. This approach allows us to reduce test data volume and scan power simultaneously. In particular, we have shown that Golomb coding of precomputed test sets leads to significant savings in peak and average power, without requiring either a slower scan clock or blocking logic in the scan cells. We have also improved upon prior work on Golomb coding by showing that a separate cyclical scan register is not necessary for pattern decompression. Experimental results for the larger ISCAS 89 benchmarks and for an IBM production circuit show that reduced test data volume and low-power scan testing can indeed be achieved in all cases.

\section{ACKNOWLEDGMENT}

The authors would like to thank B. Keller and C. Barnhart of IBM Corporation for providing scan vectors for a production circuit. They also would like to thank R. Medina for helping them in the experiments with IBM test data.

\section{REFERENCES}

[1] Y. Zorian, E. J. Marinissen, and S. Dey, "Testing embedded-core based system chips," in Proc. Int. Test Conf., 1998, pp. 130-143.

[2] Y. Zorian, "A distributed BIST control scheme for complex VLSI devices," in Proc. IEEE VLSI Test Symp., 1993, pp. 4-9.

[3] R. M. Chou, K. K. Saluja, and V. D. Agarwal, "Scheduling tests for VLSI systems under power constraints," IEEE Trans. VLSI Syst., vol. 5, pp. 175-185, June 1997.

[4] S. Wang and S. K. Gupta, "LT-RTPG: A new test-per-scan BIST TPG for low heat dissipation," in Proc. Int. Test Conf., 1999, pp. 85-94.

[5] S. Gerstendörfer and H.-J. Wunderlich, "Minimized power consumption for scan-based BIST," in Proc. Int. Test Conf., 1999, pp. 77-84.

[6] P. Girard, L. Guiller, C. Landrault, and S. Pravossoudovitch, "A test vector inhibiting technique for low energy BIST design," in Proc. IEEE VLSI Test Symp., 1999, pp. 407-412.

[7] F. Corno, M. Rebaudengo, and M. S. Reorda, "Low power BIST via nonlinear hybrid cellular automata," in Proc. IEEE VLSI Test Symp., 2000, pp. 29-34.

[8] S. Wang and S. K. Gupta, "ATPG for heat dissipation minimization during scan testing," in Proc. Design Automation Conf., 1997, pp. 614-619.

[9] V. Dabholkar, S. Chakravarty, I. Pomeranz, and S. M. Reddy, "Techniques for minimizing power dissipation in scan and combinational circuits during test application," IEEE Trans. Computer-Aided Design, vol. 17, pp. 1325-1333, Dec. 1998. 
[10] R. Sankaralingam, R. R. Oruganti, and N. A. Touba, "Static compaction techniques to control scan vector power dissipation," in Proc. IEEE VLSI Test Symp., 2000, pp. 35-40.

[11] K. Chakrabarty, "Test scheduling for core-based systems using mixedinteger linear programming," IEEE Trans. Computer-Aided Design, vol. 19 , pp. 1163-1174, Oct. 2000.

[12] M. Sugihara, H. Date, and H. Yasuura, "A novel test methodology for core-based system LSI's and a testing time minimization problem," in Proc. Int. Test Conf., 1998, pp. 465-472.

[13] K. Chakrabarty, "Design of system-on-a-chip test access architectures using integer linear programming," in Proc. IEEE VLSI Test Symp., 2000 , pp. 127-134.

[14] — "Optimal test access architectures for system-on-a-chip," ACM Trans. Design Automation Electron. Syst., vol. 6, pp. 26-49, Jan. 2001.

[15] — "Design of system-on-a-chip test access architectures under place-and-route and power constraints," in Proc. IEEE/ACM Design Automation Conf., 2000, pp. 432-437.

[16] S. Wang and S. K. Gupta, "ATPG for heat dissipation minimization during scan testing," in Proc. Design Automation Conf., 1997, pp. 614-619.

[17] V. Iyengar, K. Chakrabarty, and B. T. Murray, "Deterministic built-in pattern generation for sequential circuits," J. Electronic Testing: Theory Applicat. (JETTA), vol. 15, pp. 97-115, Aug./Oct. 1999.

[18] A. Jas, J. Ghosh-Dastidar, and N. A. Touba, "Scan vector compression/decompression using statistical coding," in Proc. IEEE VLSI Test Symp., 1999, pp. 114-120.

[19] A. Jas and N. A. Touba, "Test vector decompression via cyclical scan chains and its application to testing core-based design," in Proc. Int. Test Conf., 1998, pp. 458-464.

[20] A. Chandra and K. Chakrabarty, "Test data compression for system-on-a-chip using Golomb codes," in Proc. IEEE VLSI Test Symp., 2000, pp. 113-120.

[21] _ "System-on-a-chip test data compression and decompression architectures based on Golomb codes," IEEE Trans. Computer-Aided Design, vol. 20, pp. 355-368, Mar. 2001.

[22] — , "Test resource partitioning for SOCs," IEEE Design Test Computers, vol. 18, pp. 80-91, Sept./Oct. 2001.

[23] — - "Efficient test data compression and decompression for system-on-a-chip using internal scan chains and Golomb coding," in Proc. Design, Automation Test in Europe Conf., 2001, pp. 145-149.

[24] — "Frequency-directed run-length (FDR) codes with application to system-on-a-chip test data compression," in Proc. IEEE VLSI Test Symp., 2001, pp. 42-47.

[25] I. Hamzaoglu and J. H. Patel, "Test set compaction algorithms for combinational circuits," in Proc. Int. Conf. Computer-Aided Design, 1998, pp. 283-289.

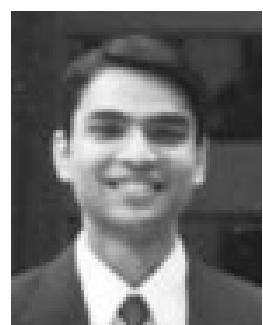

Anshuman Chandra (S'97) received the B.E. degree in electrical engineering from the University of Roorkee, Roorkee, India, in 1998, the M.S. degree in electrical and computer engineering from Duke University, Durham, NC, in 2000, and is pursuing the $\mathrm{Ph}$.D. degree at the same university.

His research interests include the fields of VLSI design, digital testing, and computer architecture. $\mathrm{He}$ is currently working in the areas of test-set compression/decompression, embedded core testing, and built-in self test.

Mr. Chandra is a student member of the ACM SIGDA. He received the Test Technology Technical Council James Beausang Student Paper Award for a paper in Proc. 2000 IEEE VLSI Test Symposium. He is also a recipient of a Best Paper Award for the 2001 Design Automation and Test in Europe (DATE) Conference.

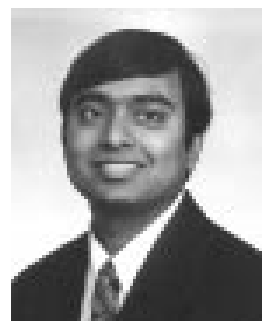

Krishnendu Chakrabarty (S'92-M'96-SM'00) received the B.Tech. degree from the Indian Institute of Technology, Kharagpur, in 1990, and the M.S.E. and $\mathrm{Ph} . \mathrm{D}$. degrees from the University of Michigan, Ann Arbor, in 1992 and 1995, respectively, all in computer science and engineering.

$\mathrm{He}$ is now Assistant Professor of Electrical and Computer Engineering at Duke University. He is also a Mercator Visiting Professor at the University of Potsdam, Germany. His current research projects include system-on-a-chip test, embedded real-time operating systems, distributed sensor networks, and architectural optimization of microelectrofluidic systems. He has published over 90 papers in archival journals and refereed conference proceedings, and he holds a U.S. patent in built-in self-test.

Dr. Chakrabarty is a recipient of the National Science Foundation Early Faculty (CAREER) Award and the Office of Naval Research Young Investigator Award. He is also a recipient of a Best Paper Award for the 2001 Design Automation and Test in Europe (DATE) Conference. He is an Associate Editor of the IEEE TRANSACTIONS ON COMPUTER-AIDED DESIGN OF INTEGRATED CIRCUITS AND SYSTEMS and an Editor of the Journal of Electronic Testing: Theory and Applications (JETTA). He is also a Guest Editor for a special issue of JETTA on system-on-a-chip testing. He is a member of ACM and ACM SIGDA, and a member of Sigma Xi. He serves as Vice Chair of Technical Activities in IEEE's Test Technology Technical Council and is a member of the program committees of several IEEE/ACM conferences and workshops. 